\title{
An Unconventional Mechanistic Insight on Aggregation Induced Emission in Novel Boron Dipyrromethenes and Their Rational Biological Realizations
}

Roop Shikha Singh ${ }^{\mathrm{a}}$, Ashish Kumar ${ }^{\mathrm{a}}$, Sujay Mukhopadhyay ${ }^{\mathrm{a}}$, Gunjan Sharma ${ }^{\mathrm{b}}$, Biplob Koch ${ }^{\mathrm{b}}$, Daya Shankar Pandey ${ }^{\mathrm{a} *}$

${ }^{a}$ Department of Chemistry, Institute of Science, Banaras Hindu University, Varanasi - 221005 (U.P.) India

${ }^{\mathrm{b}}$ Department of Zoology, Institute of Science, Banaras Hindu University, Varanasi - 221005 (U.P.) India

\section{Contents}

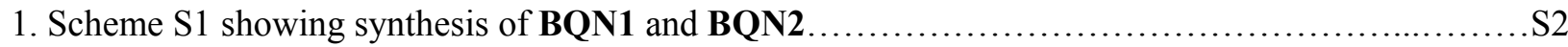

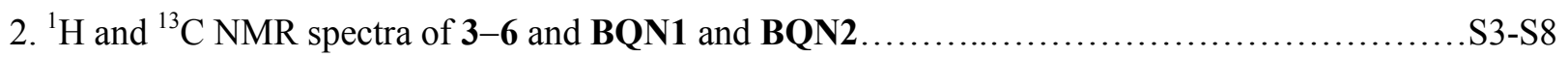

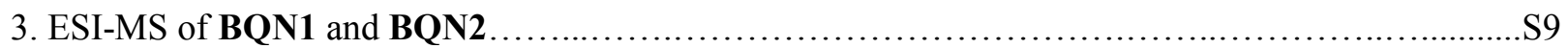

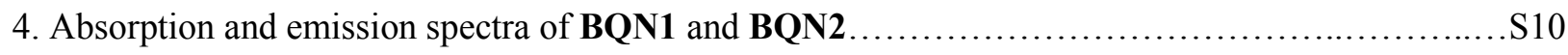

5. Absorption spectra of BQN1 and BQN2 with varying water content..................................S11

6. Time-resolved peak fluorescence of methanolic solution of BQN2 and its aggregate.....................S11

7. SEM, AFM, Fluorescence microscopic images of BQN2 loaded BSA NPs...................... S12

8. DLS spectra of BQN2 loaded BSA NPs..................................................

9. ORTEP view of BQN1 and BQN2 at 40\% ellipsoidal probability............................. 13

10. Cytotoxicity evaluation of BQN1 and BQN2 by MTT assay ....................................

11. Fluorescence images of live MDA-MB-231 cells with BQN1 and BQN2 ....................S14

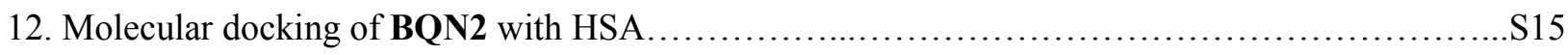

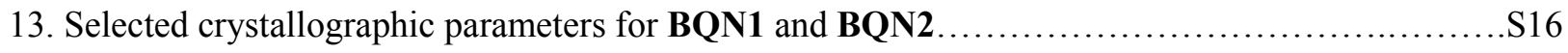

14. Selected bond distances and angles in BQN1 and BQN2 ..................................... 17 


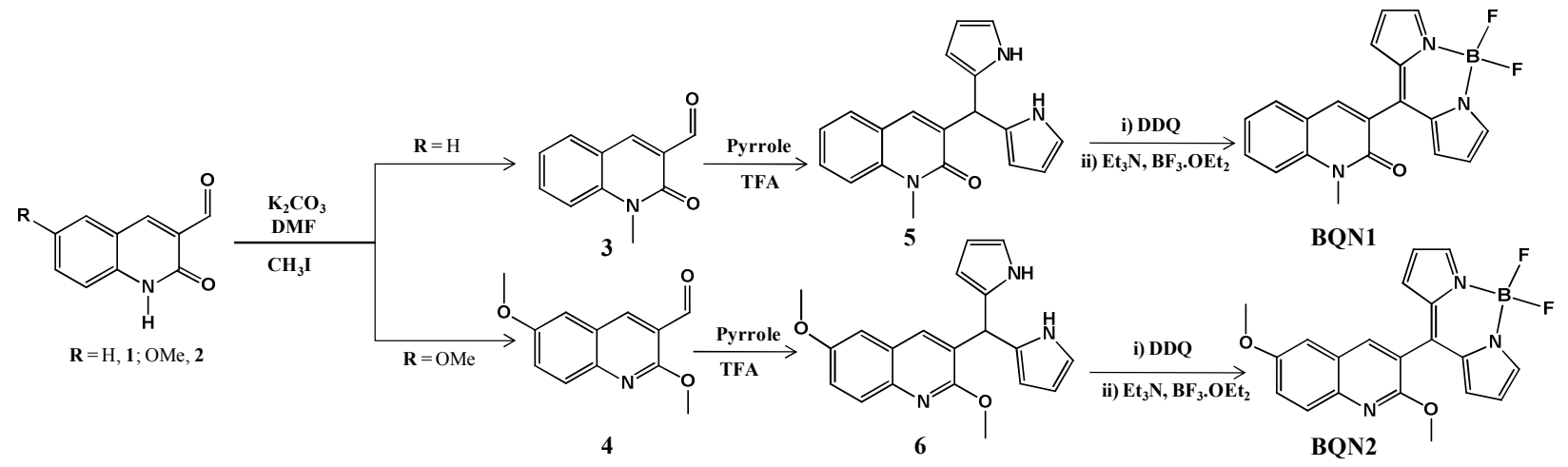

Scheme S1. Showing Synthesis of BQN1 and BQN2. 

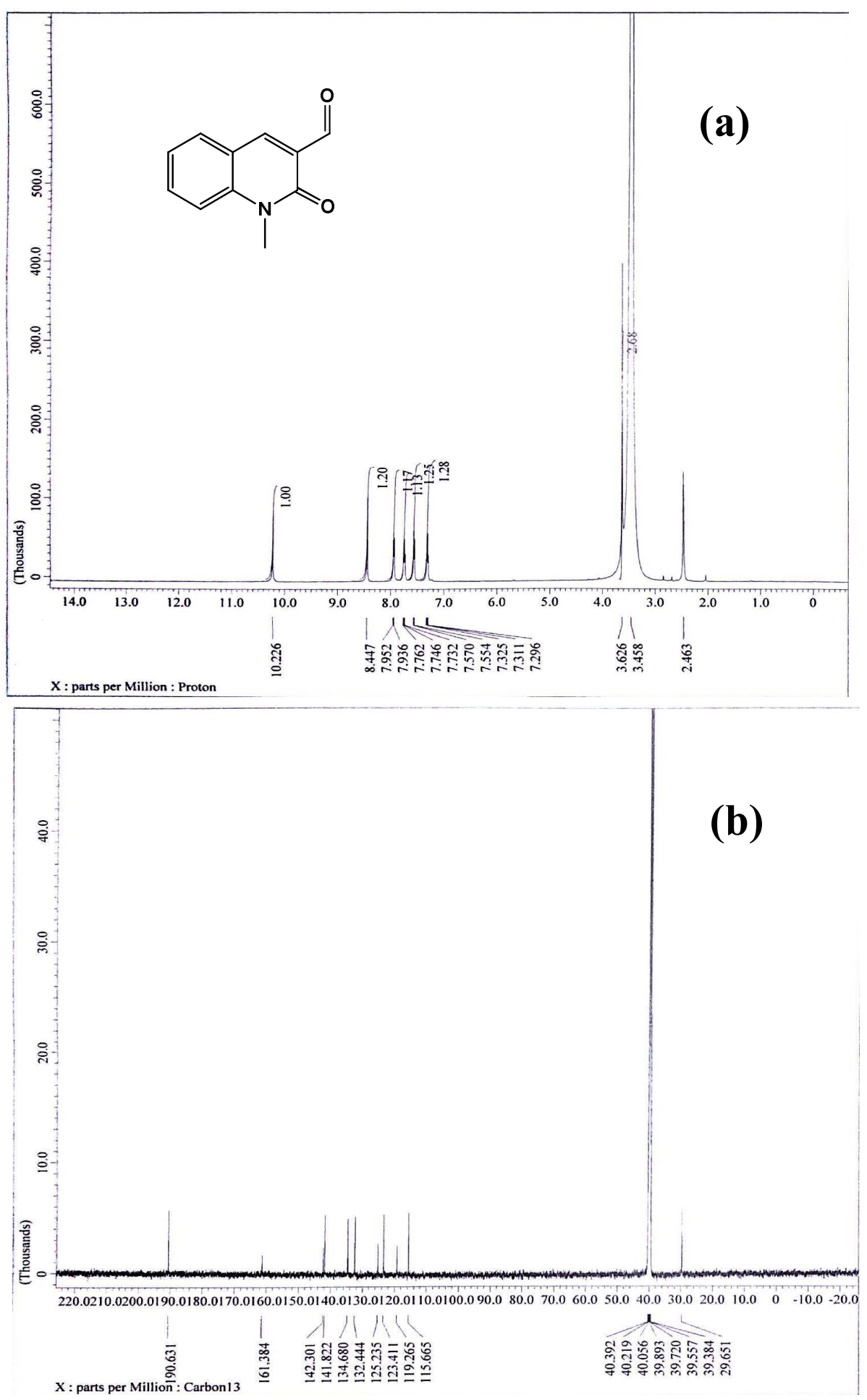

Figure S1. ${ }^{1} \mathrm{H}$ (a) and ${ }^{13} \mathrm{C}$ (b) NMR spectra of $\mathbf{3}$ in DMSO-d6. 


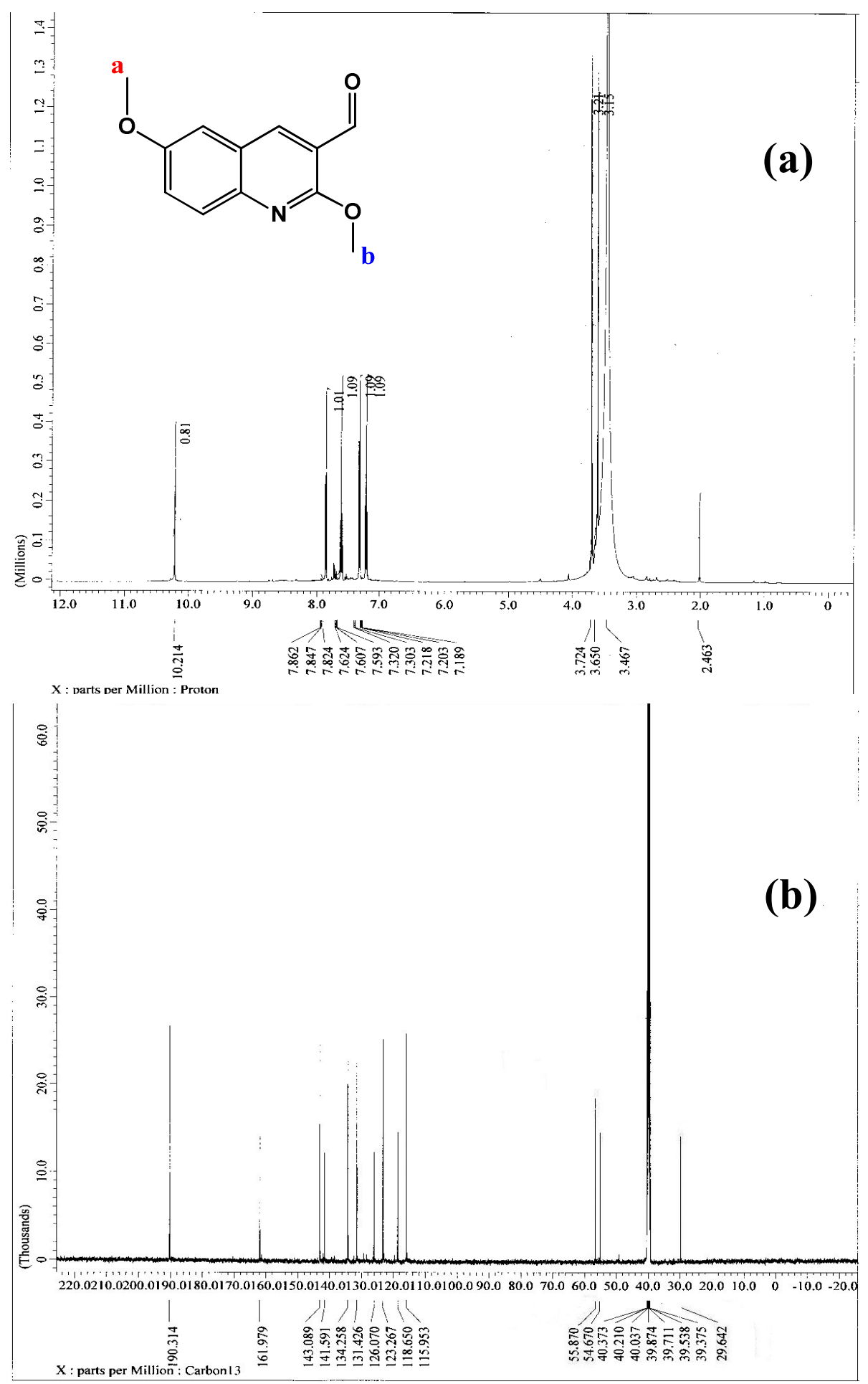

Figure S2. ${ }^{1} \mathrm{H}$ (a) and ${ }^{13} \mathrm{C}$ (b) NMR spectra of 4 in DMSO-d6. 


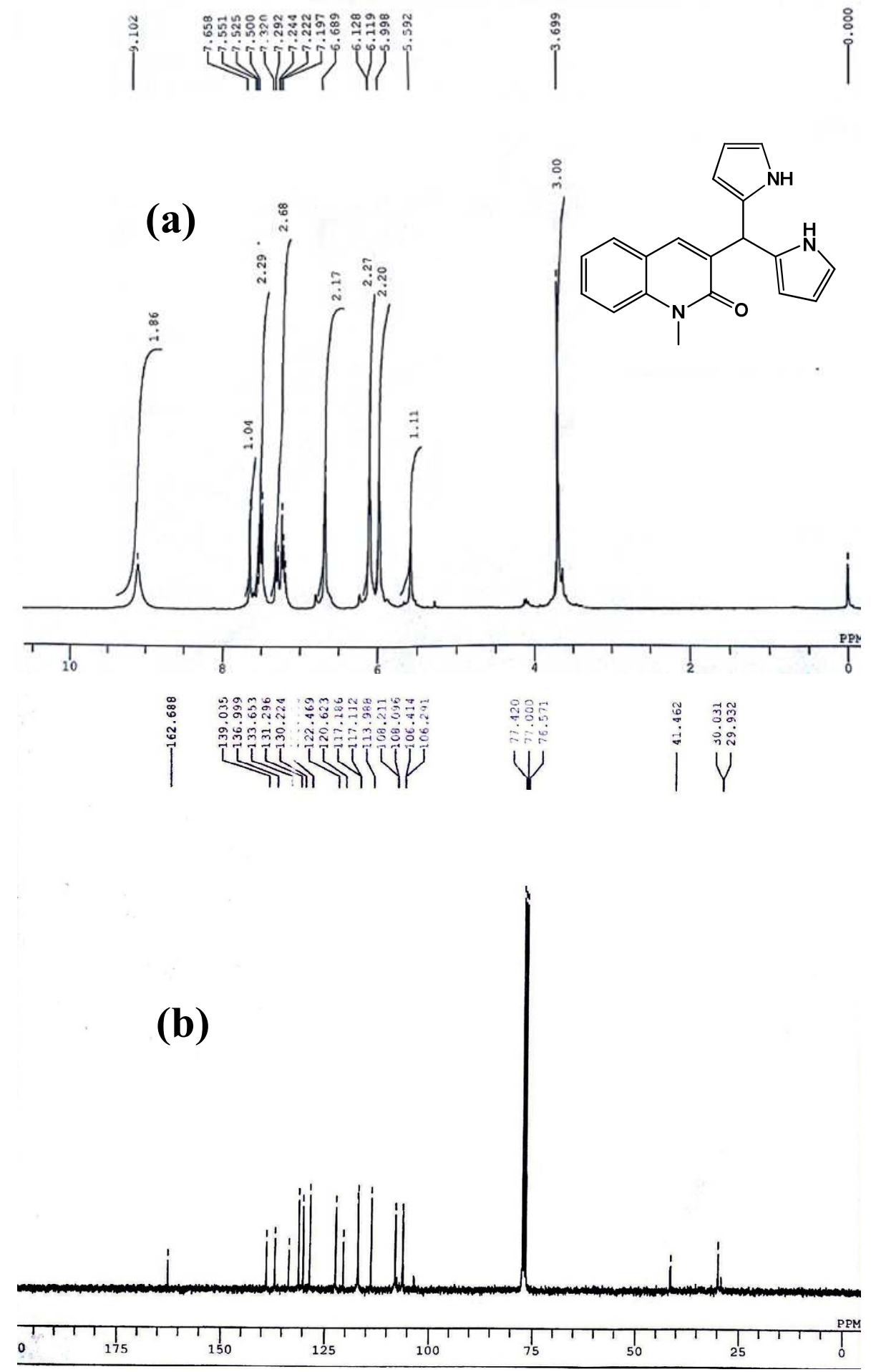

Figure S3. ${ }^{1} \mathrm{H}$ (a) and ${ }^{13} \mathrm{C}$ (b) NMR spectra of 5 in $\mathrm{CDCl}_{3}$. 

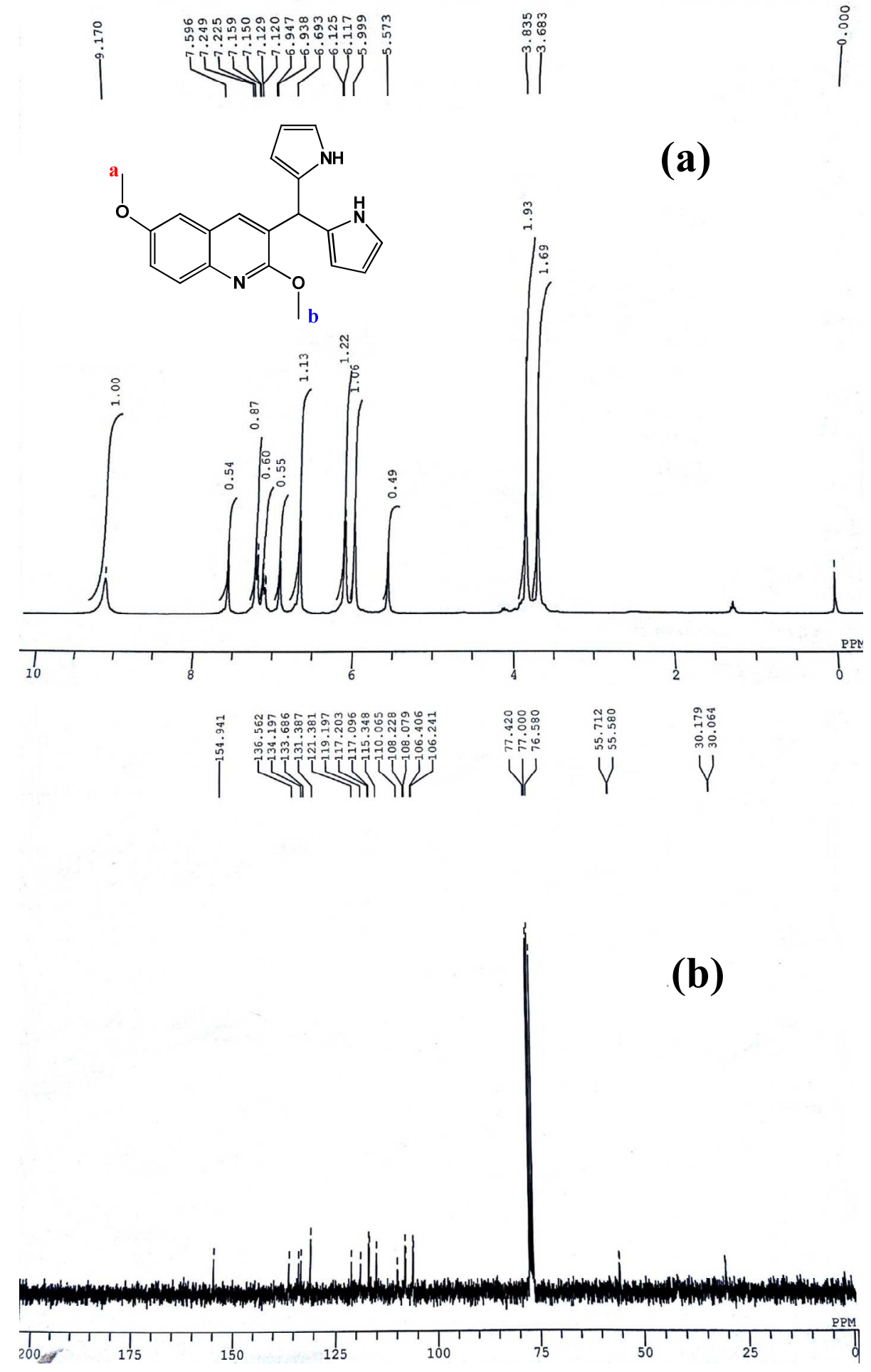

Figure S4. ${ }^{1} \mathrm{H}$ (a) and ${ }^{13} \mathrm{C}$ (b) NMR spectra of 6 in $\mathrm{CDCl}_{3}$. 

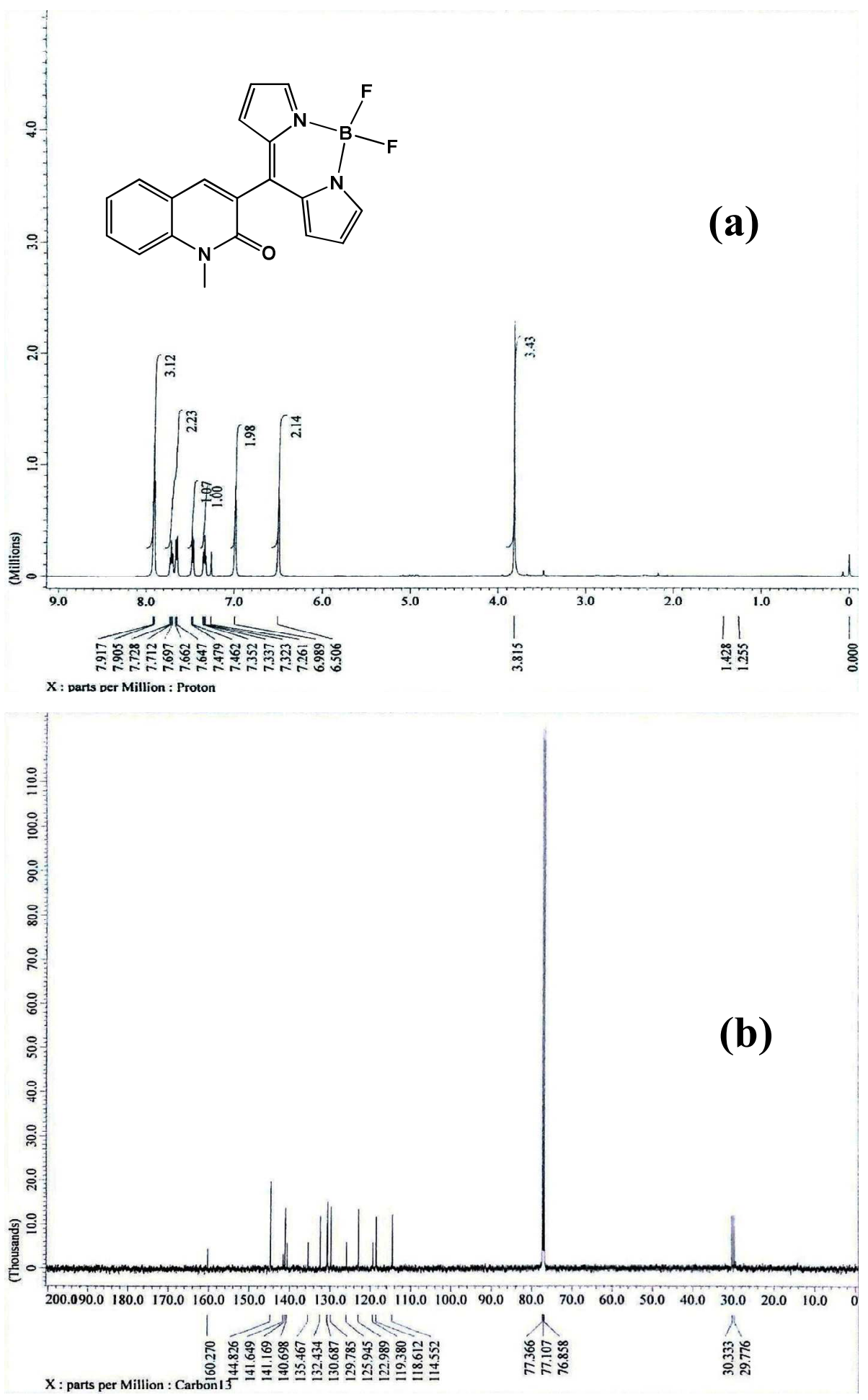

Figure S5. ${ }^{1} \mathrm{H}$ (a) and ${ }^{13} \mathrm{C}$ (b) NMR spectra of $\mathbf{B Q N 1}$ in $\mathrm{CDCl}_{3}$. 


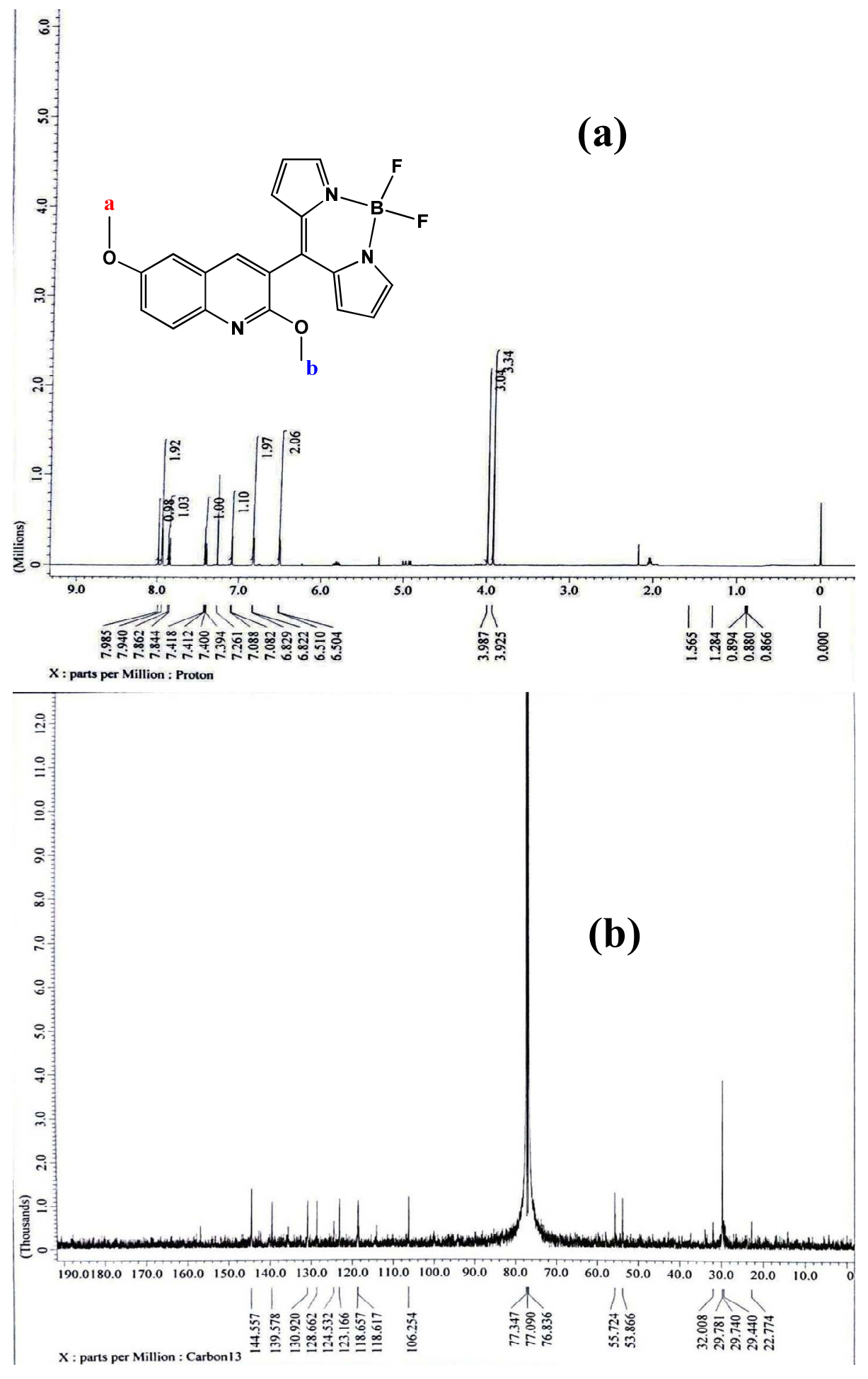

Figure S6. ${ }^{1} \mathrm{H}$ (a) and ${ }^{13} \mathrm{C}$ (b) NMR spectra of BQN2 in $\mathrm{CDCl}_{3}$. 


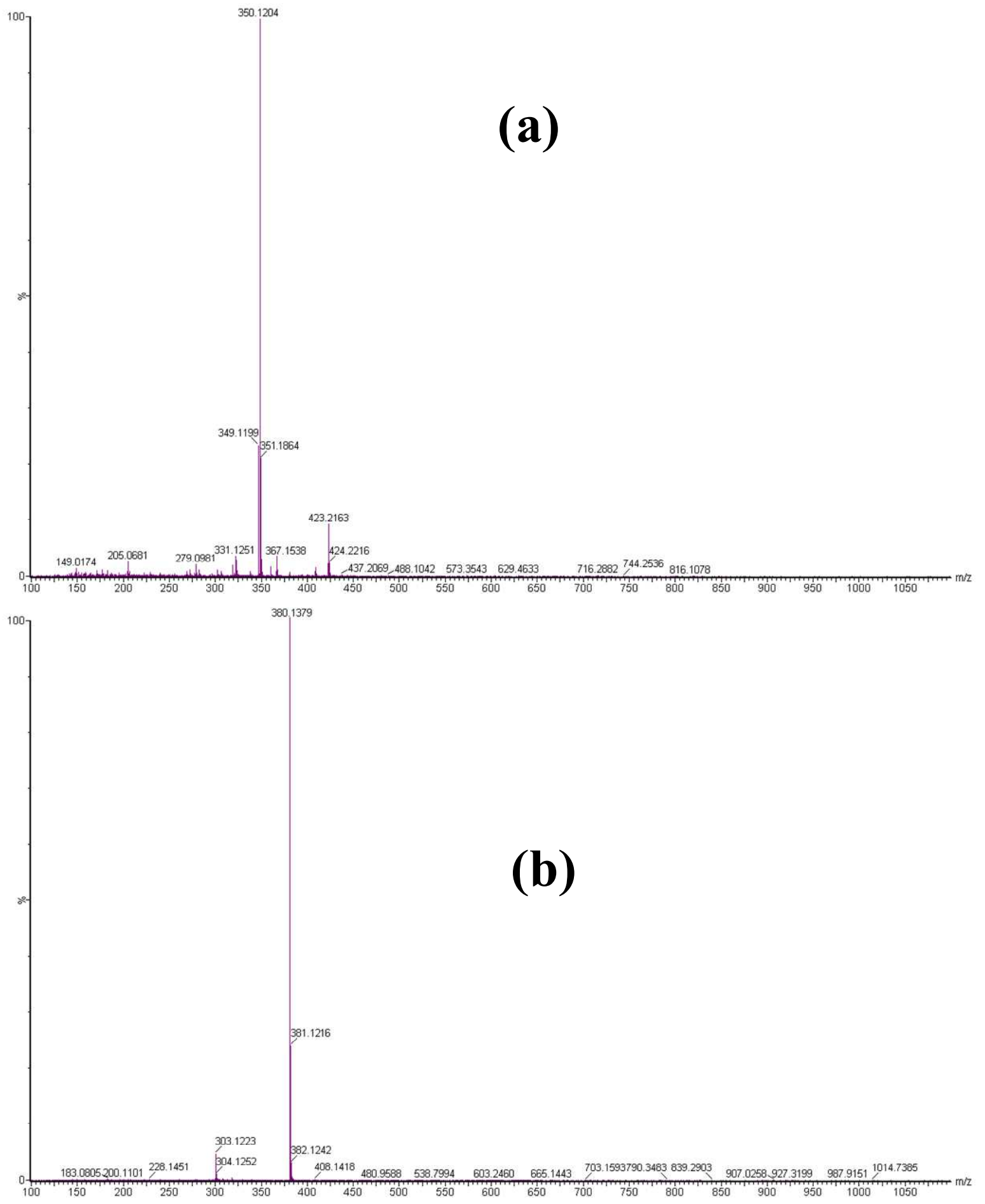

Figure S7. ESI-MS of BQN1 (a) and BQN2 (b). 

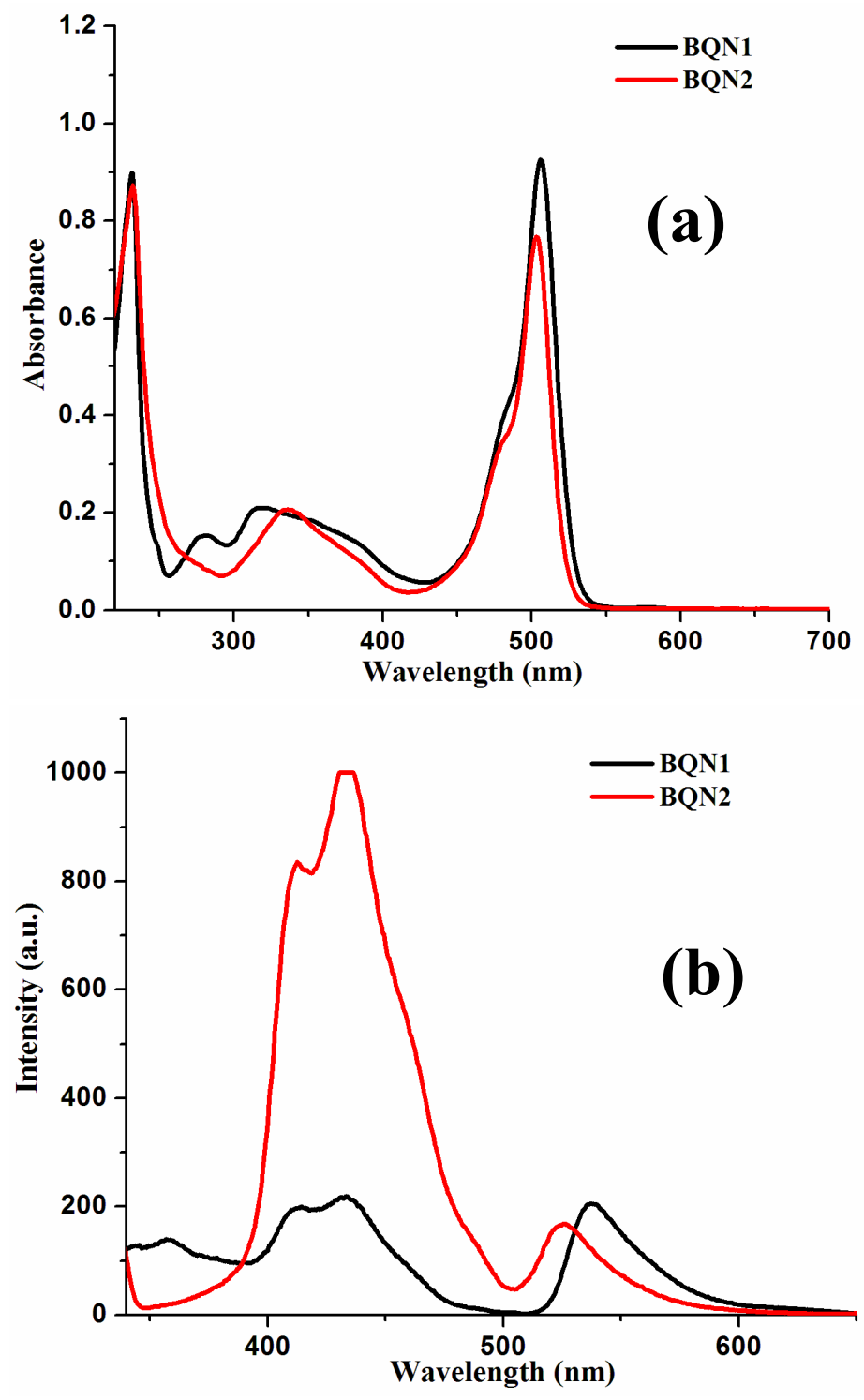

Figure S8. Absorption (a) and emission (b) spectra of BQN1 and BQN2 in methanol (c $=5 \times$ $\left.10^{-5} \mathrm{~mol} / \mathrm{L}\right)$. 

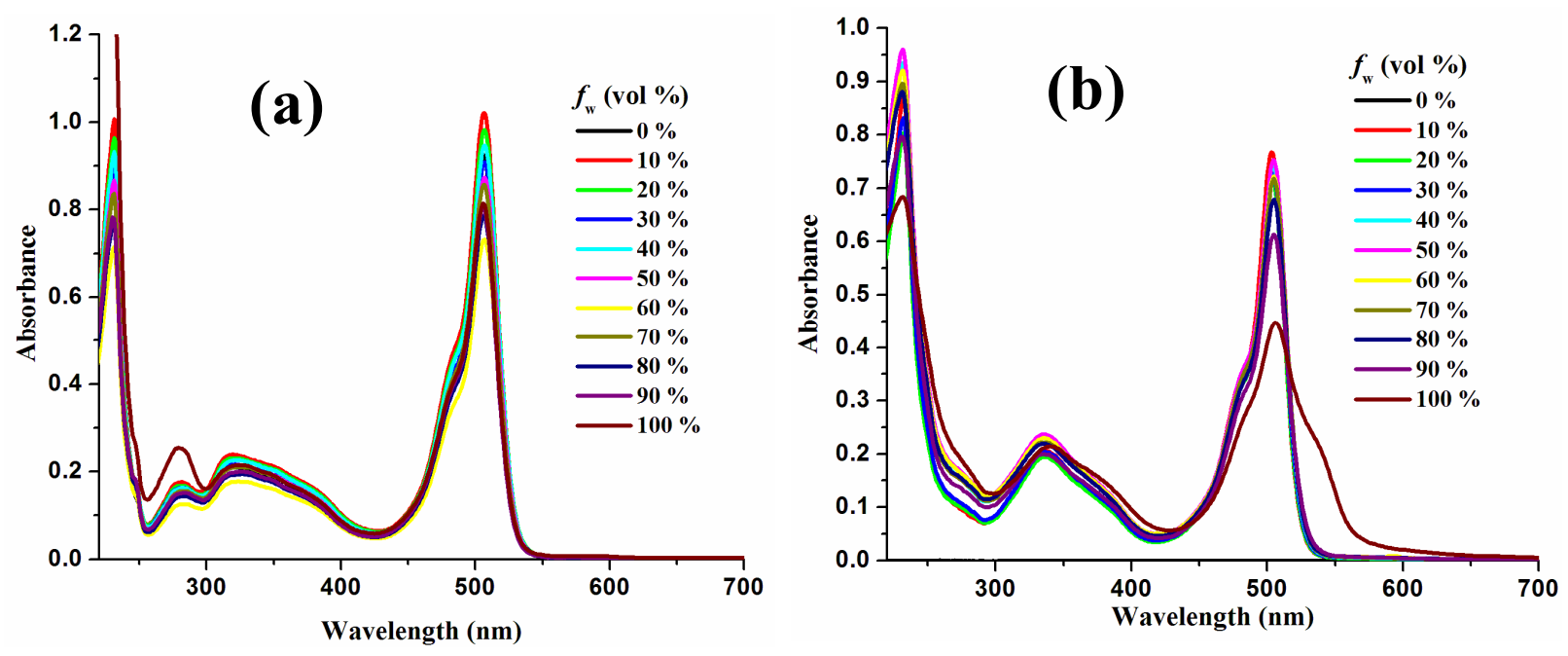

Figure S9. Absorption spectra of BQN1 (a) and BQN2 (b) in methanol/water mixtures with different water volume fractions $\left(\mathrm{c}=5 \times 10^{-5} \mathrm{~mol} / \mathrm{L}\right)$.

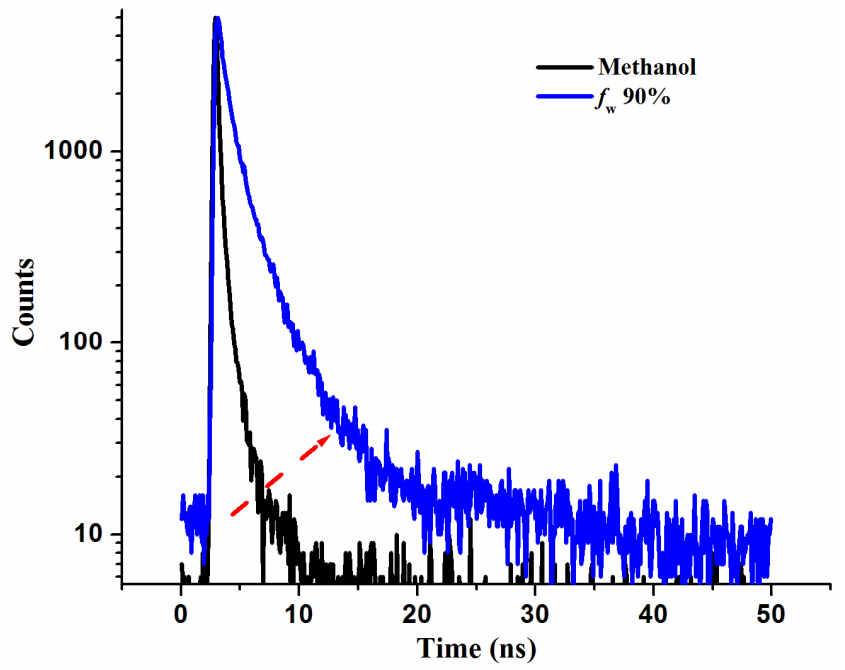

Figure S10. Time-resolved fluorescence of methanolic solution of BQN2 and its aggregate at $f_{\mathrm{w}}$ 90\%: A logarithmic view. 

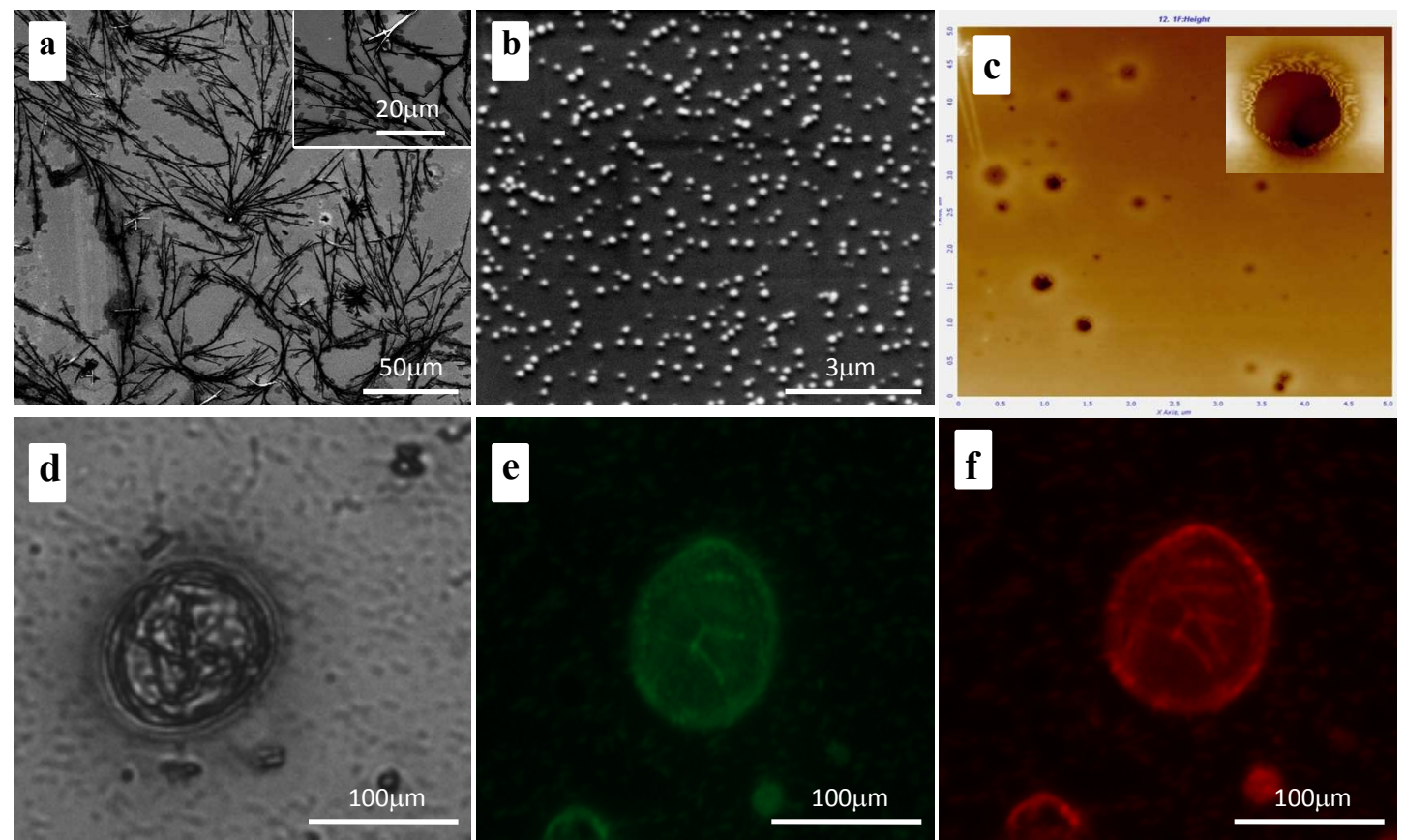

Figure S11. Figure: SEM image showing (a) initial interaction of BQN2 nanoaggregates with BSA and (b) BQN2 loaded BSA nanoparticles. AFM (c) fluorescence microscopic bright field (d) green channel (e) and red channel (f) images of BSA nanoparticle at high feeding ratio ( $>5$ $\mathrm{wt} \%)$.
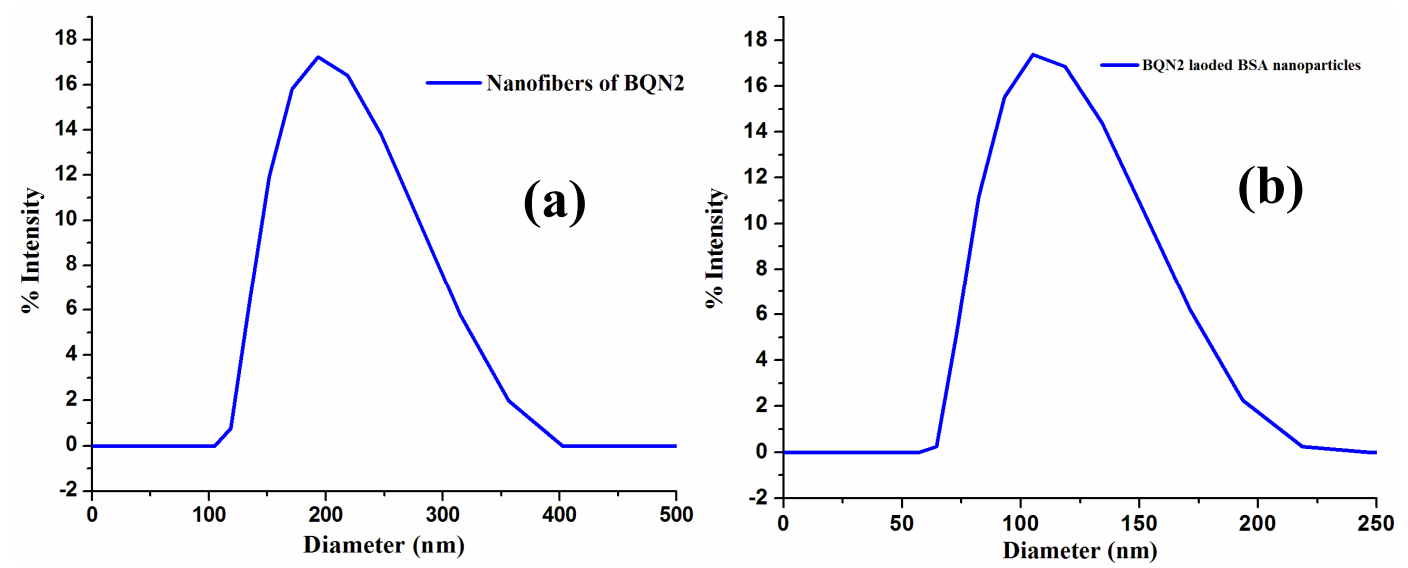

Figure S12. Size distribution obtained via DLS for nanofibers of BQN2 (a) and BQN2 loaded BSA-NPs (b). 


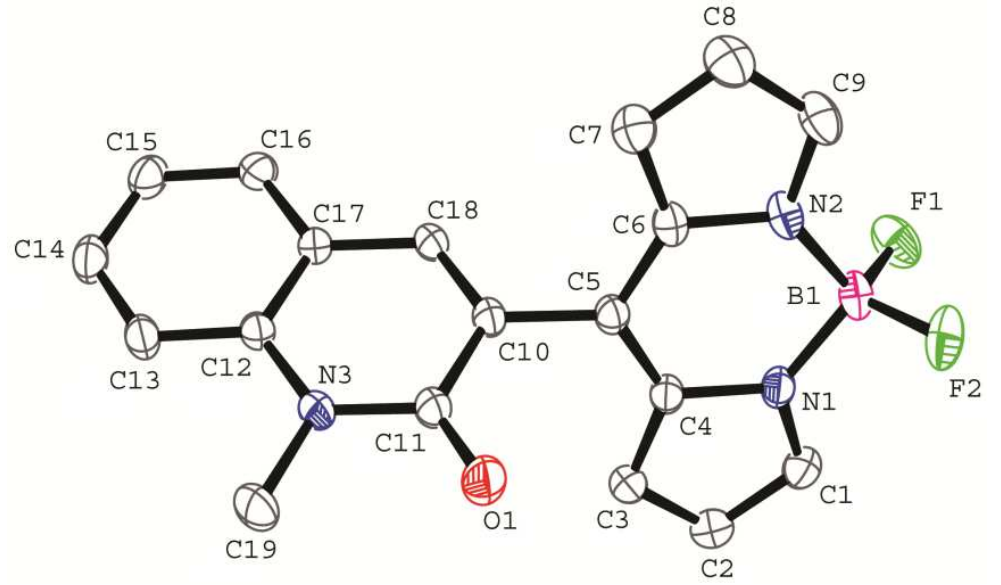

(a)

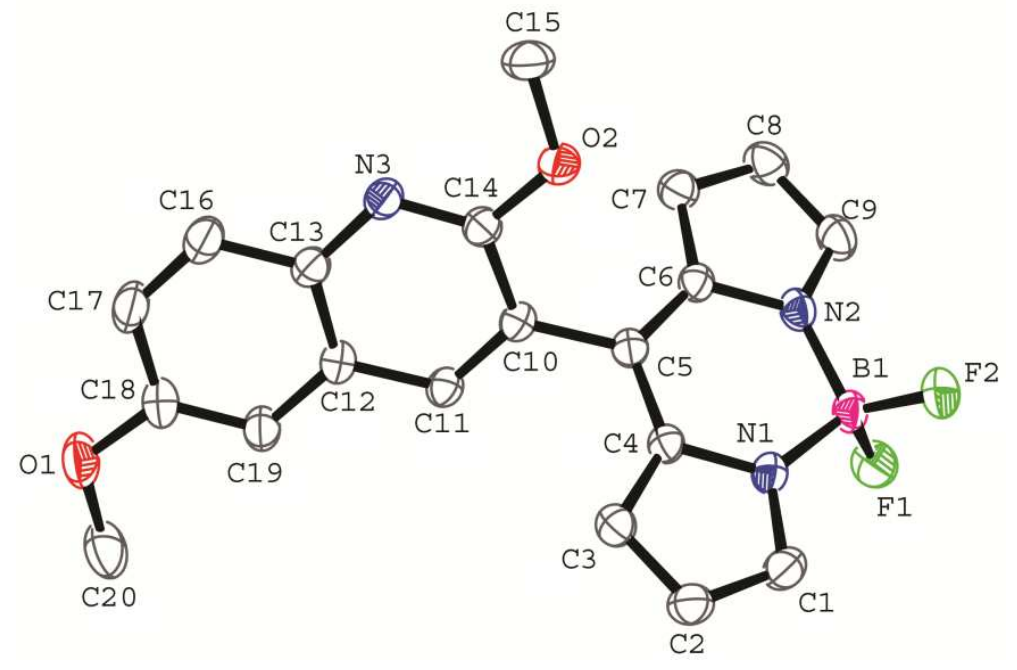

(b)

Figure S13. ORTEP view of BQN1 (a) and BQN2 (b) at 40\% ellipsoidal probability ( $\mathrm{H}$ atoms are omitted for clarity). 

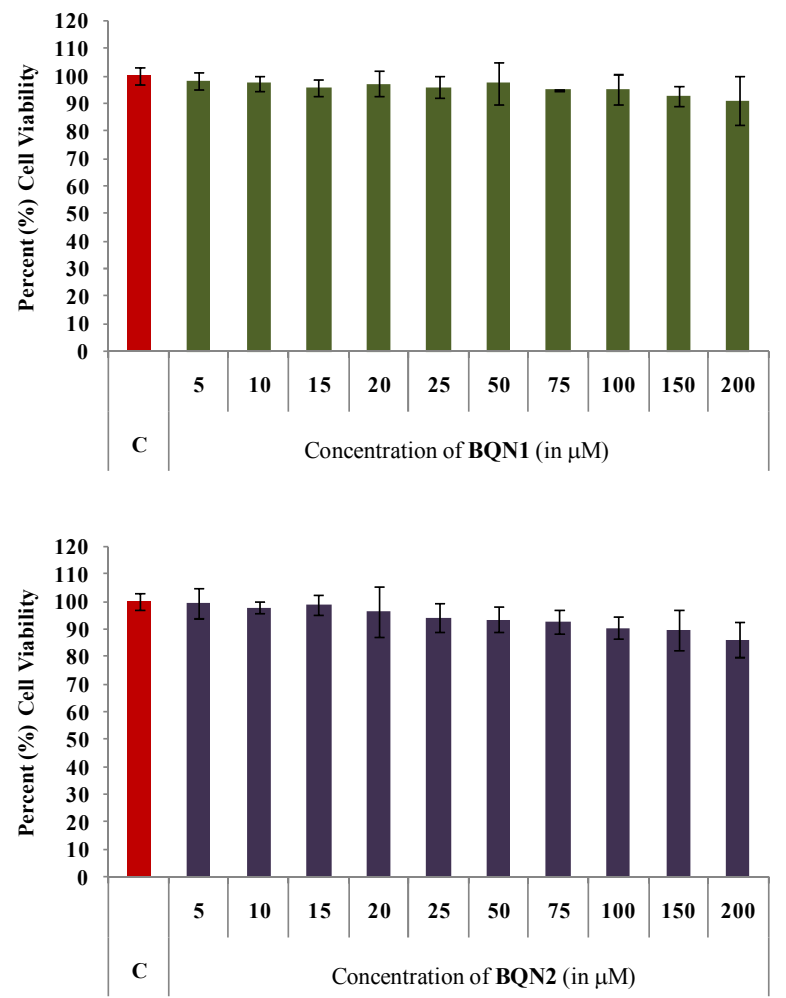

Figure S14. Cytotoxicity of BQN1 and BQN2 on human breast cancer (MDA-MB-231) cells evaluated by the MTT assay.

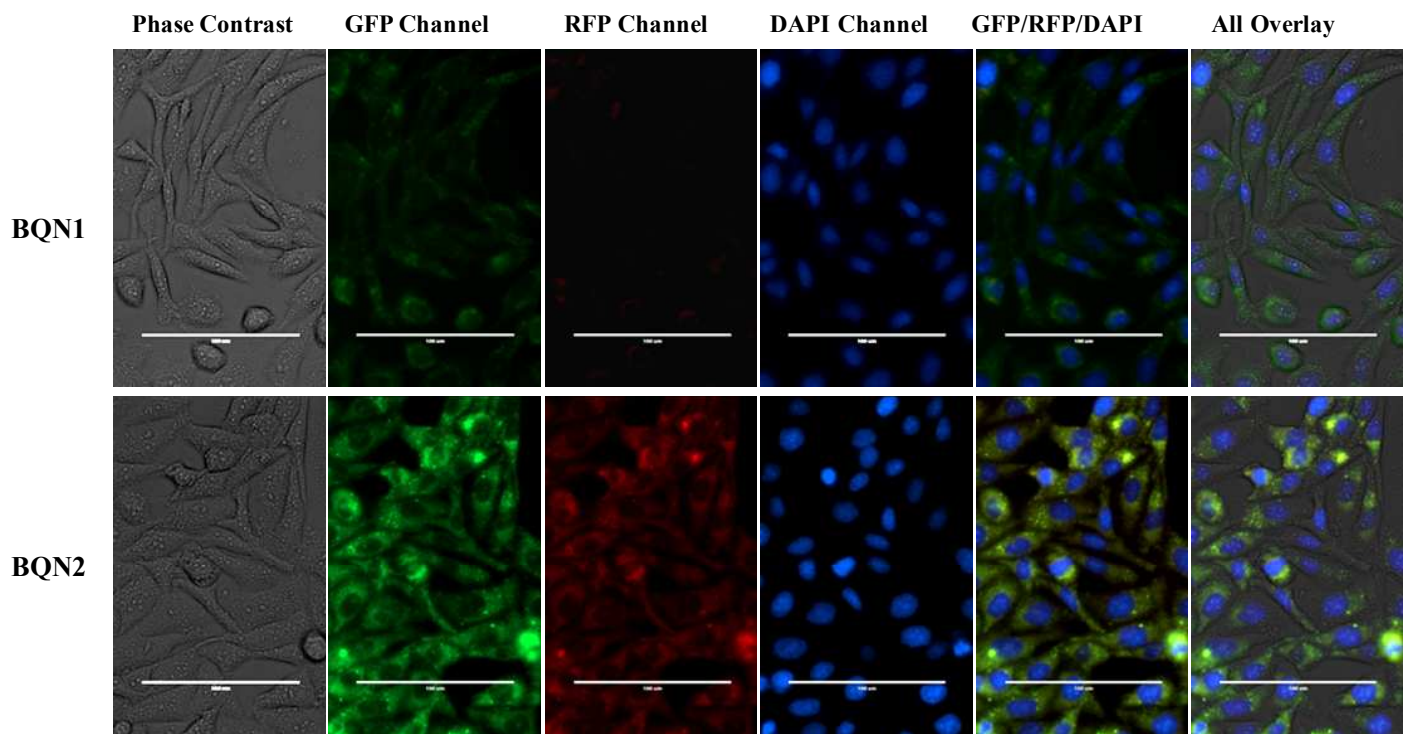

Figure S15. Fluorescence images of live MDA-MB-231 cells with complexes (BQN1 and BQN2) and co-stained with Hoechst 33342. The images from left to right show phase contrast, green channel, red channel, blue Channel, GFP/RFP/DAPI overlays and all overlays. 


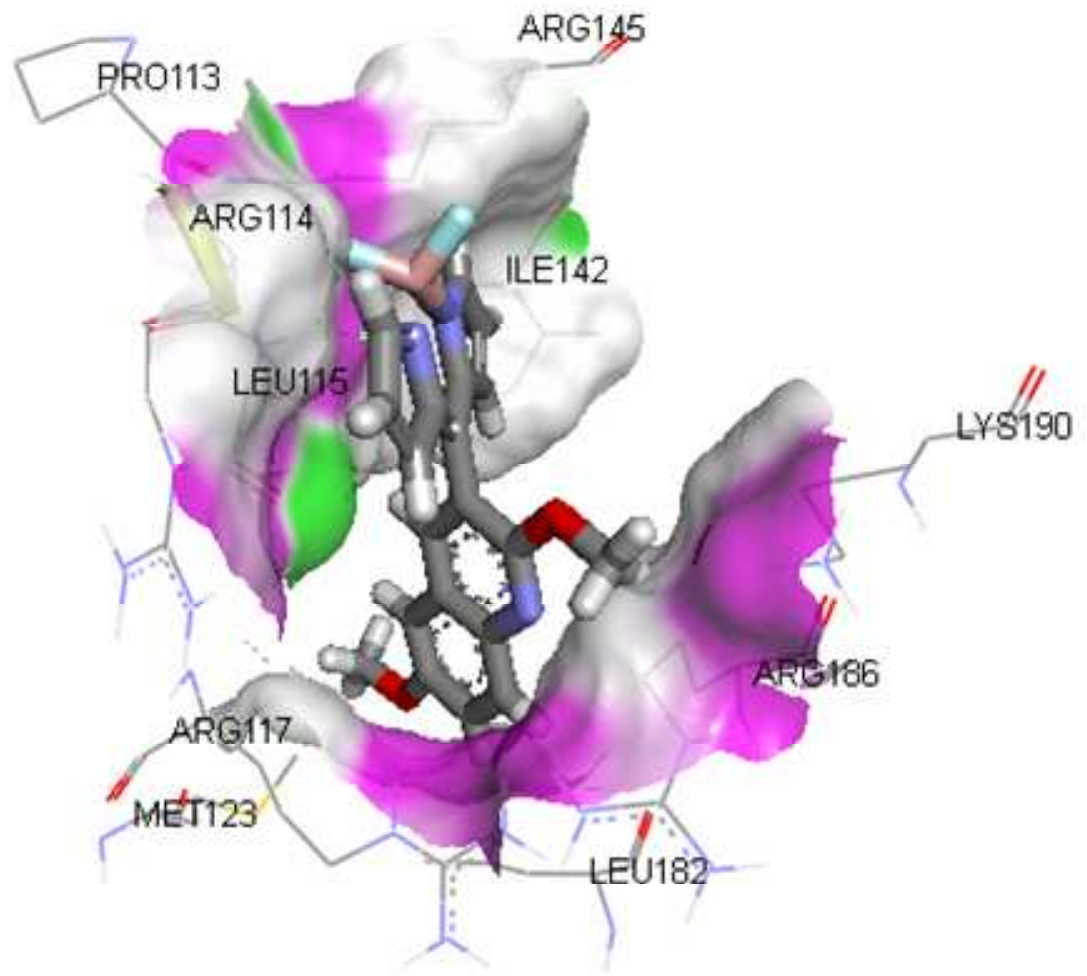

Figure S16. The interaction mode between BQN2 (stick) and HSA (cartoon): Close view. 
Table S1. Selected crystallographic parameters for BQN1 and BQN2.

\begin{tabular}{|c|c|c|}
\hline Crystal data & BQN1 & BQN2 \\
\hline Empirical formula & $\mathrm{C}_{19} \mathrm{H}_{14} \mathrm{BF}_{2} \mathrm{~N}_{3} \mathrm{O}$ & $\mathrm{C}_{20} \mathrm{H}_{16} \mathrm{BF}_{2} \mathrm{~N}_{3} \mathrm{O}_{2}$ \\
\hline Crystal system & Monoclinic & Monoclinic \\
\hline Space group & $P 2_{1} / n$ & $P 2_{1} / c$ \\
\hline$a(\AA)$ & $7.2297(2)$ & $10.4767(8)$ \\
\hline$b(\AA)$ & $9.3101(3)$ & $20.7570(11)$ \\
\hline$c(\AA)$ & $23.7779(6)$ & $8.7718(7)$ \\
\hline$\alpha\left(^{\circ}\right)$ & 90.00 & 90.00 \\
\hline$\beta\left(^{\circ}\right)$ & $96.213(2)$ & $107.202(8)$ \\
\hline$\gamma\left({ }^{\circ}\right)$ & 90.00 & 90.00 \\
\hline$V\left(\AA^{3}\right)$ & $1591.07(8)$ & $1822.2(2)$ \\
\hline $\mathrm{Z}$ & 4 & 4 \\
\hline $\mathrm{F}(000)$ & 720 & 784 \\
\hline$\rho_{\text {calc }}\left(\mathrm{Mg} \mathrm{m}^{-3}\right)$ & 1.458 & 1.382 \\
\hline$T(\mathrm{~K})$ & 293(2) & $293(2)$ \\
\hline$\mu\left(\mathrm{mm}^{-1}\right)$ & 0.108 & 0.104 \\
\hline refln collected & 5297 & 3211 \\
\hline GOF on $\mathrm{F}^{2}$ & 1.078 & 0.815 \\
\hline $\mathrm{R}$ indices (All data) & $\begin{array}{l}\mathrm{R} 1=0.0783 \\
\mathrm{wR}_{2}=0.1509\end{array}$ & $\begin{array}{l}\mathrm{R} 1=0.0849 \\
\mathrm{wR}_{2}=0.2294\end{array}$ \\
\hline final $R$ indices $[I>2 \sigma(I)]$ & $\begin{array}{l}\mathrm{R} 1=0.0553 \\
\mathrm{wR}_{2}=0.1368\end{array}$ & $\begin{array}{l}\mathrm{R} 1=0.0622 \\
\mathrm{wR}_{2}=0.2040\end{array}$ \\
\hline
\end{tabular}


Table S2. Selected bond distances (a) and angles (b) in BQN1 and BQN2.

(a)

\begin{tabular}{|c|c|c|c|}
\hline Bond Length $(\boldsymbol{\AA})$ & BQN1 & Bond Length $(\AA)$ & BQN2 \\
\hline O1-C11 & $1.229(2)$ & O2-C14 & $1.352(4)$ \\
\hline N3-C19 & $1.462(2)$ & O2-C15 & $1.431(5)$ \\
\hline N1-B1 & $1.550(2)$ & N1-B1 & $1.540(5)$ \\
\hline N2-B1 & $1.545(2)$ & N2-B1 & $1.545(5)$ \\
\hline N3-C11 & $1.3901(19)$ & N3-C14 & $1.301(4)$ \\
\hline N3-C12 & $1.394(2)$ & N3-C13 & $1.370(5)$ \\
\hline C10-C5 & $1.4894(19)$ & C10-C5 & $1.486(4)$ \\
\hline
\end{tabular}

(b)

\begin{tabular}{|c|c|c|c|}
\hline Bond Angle $\left(^{\circ}\right)$ & BQN1 & Bond Angle $\left(^{\circ}\right)$ & BQN2 \\
\hline F1-B1-F2 & $108.52(14)$ & F1-B1-F2 & $109.2(3)$ \\
\hline N2-B1-N1 & $105.93(12)$ & N2-B1-N1 & $106.1(3)$ \\
\hline C4-C5-C6 & $120.35(13)$ & C4-C5-C6 & $120.3(3)$ \\
\hline C6-C5-C10 & $119.54(14)$ & C6-C5-C10 & $120.6(3)$ \\
\hline C4-C5-C10 & $119.93(14)$ & C4-C5-C10 & $119.0(3)$ \\
\hline C11-C10-C5 & $118.92(13)$ & C11-C10-C5 & $120.8(3)$ \\
\hline C18-C10-C5 & $120.14(13)$ & C18-C10-C5 & $121.9(3)$ \\
\hline C11-N3-C19 & $116.70(14)$ & C14-O2-C15 & $117.4(3)$ \\
\hline
\end{tabular}

